\title{
A!
}

This is an electronic reprint of the original article.

This reprint may differ from the original in pagination and typographic detail.

Scarbath-Evers, Laura Katharina; Todorović, Milica; Golze, Dorothea; Hammer, René; Widdra, Wolf; Sebastiani, Daniel; Rinke, Patrick

\section{Gold diggers}

Published in:

Physical Review Materials

DOI:

10.1103/PhysRevMaterials.3.011601

Published: 17/01/2019

Document Version

Publisher's PDF, also known as Version of record

Please cite the original version:

Scarbath-Evers, L. K., Todorovi, M., Golze, D., Hammer, R., Widdra, W., Sebastiani, D., \& Rinke, P. (2019). Gold diggers: Altered reconstruction of the gold surface by physisorbed aromatic oligomers. Physical Review Materials, 3(1), 1-6. [011601]. https://doi.org/10.1103/PhysRevMaterials.3.011601

This material is protected by copyright and other intellectual property rights, and duplication or sale of all or part of any of the repository collections is not permitted, except that material may be duplicated by you for your research use or educational purposes in electronic or print form. You must obtain permission for any other use. Electronic or print copies may not be offered, whether for sale or otherwise to anyone who is not an authorised user. 


\title{
Gold diggers: Altered reconstruction of the gold surface by physisorbed aromatic oligomers
}

\author{
Laura Katharina Scarbath-Evers, ${ }^{1, *}$ Milica Todorović, ${ }^{2}$ Dorothea Golze, ${ }^{2}$ René Hammer, ${ }^{3}$ Wolf Widdra, ${ }^{3}$ \\ Daniel Sebastiani, ${ }^{1}$ and Patrick Rinke ${ }^{2}$ \\ ${ }^{1}$ Institute of Chemistry, Martin-Luther University Halle-Wittenberg, Halle, Germany \\ ${ }^{2}$ Department of Applied Physics, Aalto University School of Science, FI-00076 Aalto, Finland \\ ${ }^{3}$ Institute of Physics, Martin-Luther University Halle-Wittenberg, Halle, Germany
}

(Received 7 October 2018; revised manuscript received 26 November 2018; published 17 January 2019)

\begin{abstract}
We present electronic structure theory calculations and scanning tunneling microscopy experiments for the adsorption of $\alpha$-sexithiophene on the 100 surface of gold. Our density-functional theory calculations show that $\alpha$-sexithiophene prefers to adjust an energetically unfavorable adsorption site by modifying the gold surface over seeking out more favorable adsorption sites. Molecular adsorption results in a complex charge transfer pattern, with more charge transfer in more stable sites. Our results challenge the current paradigm that weakly interacting (e.g., physisorbed) molecules perceive metal surfaces as rigid templates with preordained adsorption sites.
\end{abstract}

DOI: 10.1103/PhysRevMaterials.3.011601

Gold has a wide range of industrial applications, from catalyzing oxidation reactions [1-5] to electrodes in organic field-emitting transistors. This versatility has fueled interest in the properties of organic-gold interfaces [6-15]. What makes the low-index surfaces of gold $(\mathrm{Au}(100)$ [16-18], $\mathrm{Au}(110)$ $[19,20]$, and $\mathrm{Au}(111)$ [21,22]) special are their large-scale reconstructions that all have characteristic height modulations in their top layer and offer various adsorption sites for organic molecules of different adsorption strengths. These characteristic patterns could be used as templates to guide molecular assembly $[10,11]$. This patterning concept assumes that distinct adsorption sites exist on these surfaces, that organic molecules will migrate almost exclusively to energetically preferred adsorption sites, and that the molecule-surface interaction does not affect the reconstruction used for patterning. Molecules that interact strongly with the gold surface violate at least one of these assumptions and the patterning concept breaks down [6-9,23-25]. In this Rapid Communication, we show that, contrary to common expectations, also weakly interacting molecules violate the patterning conditions. For the example of $\alpha$-sexithiophene on the $\mathrm{Au}(100)$ surface, we show by means of density-functional theory (DFT) calculations a surprising, barrier-free reconstruction of the ridge site into the valley site. $\alpha$-sexithiophene ( $\alpha-6 \mathrm{~T})$, depicted in Fig. 1(a), is a prototypical hole conducting organic semiconductor [26-28] that serves as a model system for polythiophenes and has already been employed successfully in optoelectronic devices such as thin-film transistors [29-33].

The reconstructed $\mathrm{Au}(100)$ surface exhibits a contracted, quasihexagonal top layer with reconstruction rows running along the [110] direction. The resulting complex height modulation of the topmost layer gives rise to a variety of different adsorption sites that can be broadly classified according to their height into on ridge and in valley, with an energetic preference for the latter. For weak molecular adsorption, one

*laura.scarbath-evers@chemie.uni-halle.de might therefore expect that a molecule placed in an unfavorable on-ridge site would migrate into a more favorable valley site, as schematically shown in scenario A in Fig. 1(b). What we find instead is that the molecule descends vertically and displaces the gold ridge, as depicted in scenario B in Fig. 1(b). This behavior is incompatible with the concept of well-defined adsorption sites and forces us to reconsider patterning strategies for organic films on metal substrates.

In the following, we present and describe our DFT calculations and scanning tunneling microscopy (STM) experiments and discuss our results. We then rationalize the behavior of $\alpha$ sexithiophene on $\mathrm{Au}(100)$ and put our results into the context of organic-inorganic interfaces.

All STM measurements have been performed in ultrahigh vacuum conditions. $\mathrm{Au}(100)$ sample preparation by $\mathrm{Ar}^{+}$ sputtering and annealing cycles followed the procedure in Ref. [18]. STM and low-energy electron diffraction experiments reveal the $c(28 \times 48) \mathrm{Au}(100)$ reconstruction prior to $\alpha$-6T evaporation onto the surface at $300 \mathrm{~K}$.

Calculations were carried out with the electronic structure code FHI-AIMS [34] at the DFT level. All adsorption structures were obtained by performing geometry optimizations in which the structure was relaxed until forces were smaller than $10^{-3} \mathrm{eV} \AA^{-1}$. We used the tier 1-tight numerical basis sets, the Perdew-Burke-Ernzerhof (PBE) exchange-correlation functional [35], the Tkatchenko-Scheffler van der Waals correction (TS vdW) [36] (for more details, please see the Supplemental Material [37]), and the atomic zeroth-order regular approximation (ZORA) $[38,39]$ to account for relativistic effects.

Since the experimentally observed $c(28 \times 48)$ supercell of the bare $\mathrm{Au}(100)$ surface is computationally not tractable, we chose the $20 \times 5$ model developed by Havu et al. [40]. With unit cell vectors of $|a|=58.97 \AA$ and $|b|=15.8 \AA$ this model is computationally still feasible while retaining the main physical features of the surface such as two-dimensional lateral contraction, rotation, and differently buckled qualitative surface areas $[16,41,42]$. Our slab consists of five layers of gold of which the three lowest were kept fixed during structural 


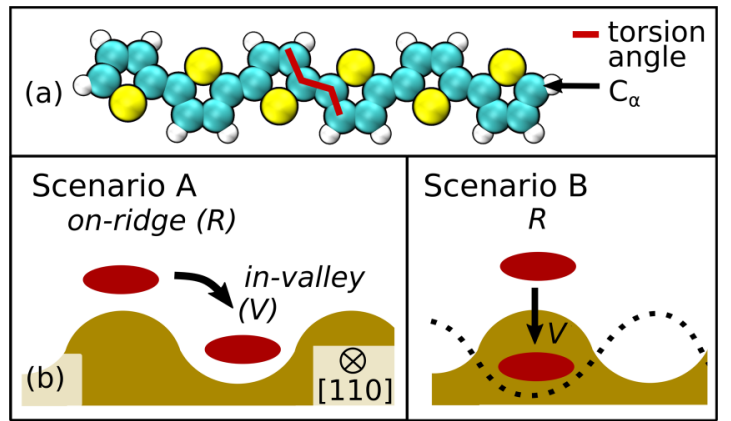

FIG. 1. (a) Sketch of $\alpha$-sexithiophene ( $\alpha-6 \mathrm{~T})$. Carbon atoms bonded to sulfur atoms are denoted as $\mathrm{C}_{\alpha}$. The planarity of the molecule can be characterized by the five torsion angles between the thiophene units, as indicated in red for the central thiophene units. (b) Adsorption schematic of $\alpha-6 \mathrm{~T}$ on the reconstructed $\mathrm{Au}(100)$ surface. In scenario A, the molecule migrates into a valley when initially placed on an ill-favored on-ridge position. In scenario B, the molecule digs itself into its preferred in-valley site by pushing the ridge aside.

relaxation. A $1 \times 2 \times 1 k$-point grid and periodic boundary conditions were employed for the surface slab using a bulk lattice constant of $4.150 \AA$. The latter has been optimized at the PBE level using a $10 \times 10 \times 10 k$-point grid per bulk unit cell.

An overview of the structure of the clean, reconstructed $\mathrm{Au}(100)$ surface is represented in Fig. 2(a). As visible in the STM image, the reconstruction rows with an apparent height of approximately $0.6 \AA$ are oriented parallel to the [110] direction. Perpendicular to the reconstruction rows, elongated areas of slight depressions can be perceived that give rise to a rhombic lattice with a $c(28 \times 48)$ supercell [18] which is indicated by the blue rectangle. Due to this complex pattern, various different adsorption sites exist that can be broadly classified according to their height into on ridge and in valley of the reconstruction rows in either the dark or bright areas of the rhombic pattern. A color-coded height image of the $20 \times 5$ DFT-model surface is presented in Fig. 2(b) with the ridges of the reconstruction rows depicted in white and the reconstruction valleys depicted in brown. The computed maximum corrugation height for the clean surface is $0.67 \AA$, in good agreement with experimental findings [18].

In the following, we describe how molecular adsorption affects the corrugation of the $\mathrm{Au}(100)$ surface. We investigate the two different adsorption environments on ridge $(r)$ and in valley (v) by optimizing the structure of an $\alpha-6 \mathrm{~T}$ molecule placed either on top or in the valley of a reconstruction row. Figure 2(c) depicts the color-coded height image of the $\mathrm{Au}(100)$ surface after adsorption when the molecule was initially placed in a valley. Compared to the clean surface, the adsorption amplifies the maximum corrugation height to $0.77 \AA$ but does not change the overall reconstruction. The corrugation pattern shows that the molecular adsorption makes the valley slightly asymmetric, as indicated by the different heights of the adjacent gold atoms on the slope depicted in the height profile in Fig. 2(i).

For on-ridge adsorption we expected the molecule to slide down into the valley, as depicted in scenario A in Fig. 1(b). Surprisingly, we found scenario B, in which the adsorbed molecule induced an inversion of the surface reconstruction pattern by pushing the reconstruction ridge aside and creating its own in-valley environment at the original on-ridge adsorp-

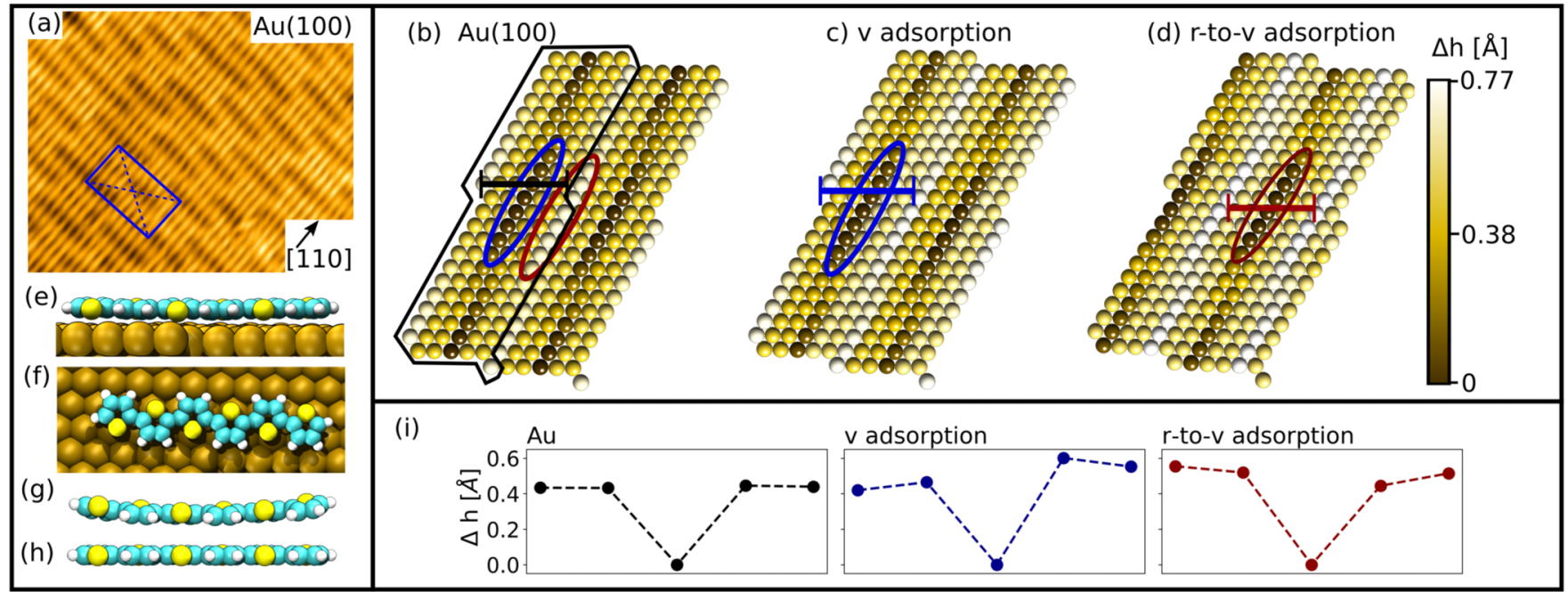

FIG. 2. (a) STM image of the clean $\mathrm{Au}(100)$ surface $(100 \mathrm{pA}, 0.5 \mathrm{~V}, 80 \mathrm{~K})$. The blue rectangle depicts the unit cell of the reconstructed $\mathrm{Au}(100)$ surface [18]. The height variation between the valleys (dark brown) and the ridges (white) corresponds to $0.6 \AA$. (b)-(d) Color-coded $1 \times 2$ supercells of the clean model surface (b) and the surface after in-valley (v) adsorption (c) and on-ridge (r) adsorption (d). The investigated positions are encircled in red and blue. Adsorption of the molecule on the ill-favored $r$ position induces a translation of the reconstruction row and the formation of a reconstruction valley directly underneath the molecule (r-to-v). (i) Height profiles across the reconstruction valleys (indicated by a bar in (b)-(d)). The reconstruction valley created by the molecule after r-to-v adsorption exhibits a higher symmetry than the reconstruction valley after $\mathrm{v}$ adsorption. (e) and (f) Structure of the molecule adsorbed in valley. The molecule adsorbs completely flat with the $\mathrm{C}_{\alpha}$ atoms above the valley (e) and, compared to the gas phase molecule depicted in ( $\mathrm{g}$ ), exhibits a considerably more planar structure that resembles the one of the cationic $\alpha-6 \mathrm{~T}$, depicted in (h). 
TABLE I. Median adsorption height $(\Delta h)$ between $\mathrm{S}$ and Au atoms, median torsion angle (the median torsional angle of the gas phase molecule is $165^{\circ}$ ), adsorption energy $E_{\text {ads }}$, and charge transfer $\delta \mathrm{Q}$ for $\alpha-6 \mathrm{~T}$ adsorbed on the reconstructed $\mathrm{Au}$ surface. For the adsorption positions v-fix and r-fix, the gold atoms were kept fixed during DFT geometry optimization. The calculated charge transfer is based on a Mulliken population analysis [43].

\begin{tabular}{lcccc}
\hline \hline Site & $\Delta h(\AA)$ & $\phi_{\text {med }}(\mathrm{deg})$ & $E_{\text {ads }}(\mathrm{eV})$ & $\delta Q\left(e^{-}\right)$ \\
\hline v & 3.25 & 179 & 4.34 & 0.44 \\
r-to-v & 3.18 & 177 & 4.31 & 0.45 \\
v-fix & 3.31 & 177 & 4.23 & 0.40 \\
r-fix & 3.47 & 168 & 3.79 & 0.32 \\
\hline \hline
\end{tabular}

tion site. Figure 2(d) shows the color-coded corrugation of the surface after adsorption of the molecule at the $r$ position, with the adsorption position of the molecule indicated in red. It can be seen that the surface now features a valley at the adsorption position. Conversely, the initial valley on the surface has turned into a reconstruction ridge. In the following, we will denote this induced valley-like adsorption environment the r-to-v position.

Figure 2(i) depicts height profiles of the bare surface and the surface after adsorption on the $\mathrm{v}$ position and the r-to- $\mathrm{v}$ position. Despite their considerable similarity, the $\mathrm{v}$ position and the r-to-v position are not identical. After v adsorption, the reconstruction valley exhibits a higher asymmetry as the r-to- $\mathrm{V}$ position as manifested in different heights of the adjacent gold atoms that form the slope of the reconstruction row. However, due to the shallow potential energy surface [40] of the reconstructed gold surface, their adsorption energies are almost indistinguishable, differing by only $0.03 \mathrm{eV}$ (see Table I). The malleability of the gold surface gives rise to a plethora of adsorption environments without distinct and clearly identifiable adsorption sites. That absence of welldefined adsorption sites on the $\mathrm{Au}(100)$ surface connects to theoretical studies regarding $\mathrm{CO}$ adsorption on small $\mathrm{Au}$ clusters that observed a dynamical reordering of surface gold atoms [44].

After characterizing the impact of the molecule on the surface structure, we subsequently address the molecular structure upon adsorption in terms of adsorption height, median torsion angle, and charge transfer, as summarized in Table I. Following the almost equal adsorption energies in the $\mathrm{v}$ position and the r-to-v position, $\alpha-6 \mathrm{~T}$ exhibits a completely flat adsorption geometry in both positions, as represented in Fig. 2(e) for the v position. Sulfur atoms are found mostly on top of gold atoms with a median adsorption height between the sulfur and the gold atoms of $3.25 \AA$ for the v position and $3.18 \AA$ for the r-to- $v$ position. The more planar adsorption geometry compared to $\alpha-6 \mathrm{~T}$ in the gas phase is also manifested in the larger torsion angles of $179^{\circ}$ on the v position and $177^{\circ}$ on the r-to-v position compared to $165^{\circ}$ for $\alpha-6 \mathrm{~T}$ in the gas phase. Such a planar structure is characteristic for the singly charged $\alpha-6 \mathrm{~T}$ molecule, depicted in Fig. 2(h), and suggests an adsorption-induced charge transfer. This assumption is confirmed by calculations of the Mulliken charges before and after adsorption that reveal a charge transfer from the molecule to the surface for both adsorption positions. The magnitude of the charge transfer is $0.44 e^{-}$and $0.45 e^{-}$for the $\mathrm{v}$ position and the r-to- $\mathrm{v}$ position, respectively.

Next, we address the question why moving many gold atoms out of the way is energetically cheaper for the molecule than sliding down the ridge into the valley. To find the real adiabatic pathways for either process would require complex minimum energy path (MEP) calculations [45], which are computationally not tractable for large systems such as ours. We therefore approximate the pathways by performing a linear interpolation between the coordinates of the starting and the final structure for scenarios A and B, respectively. For scenario $\mathrm{B}$, in which the molecule digs itself in, we take the starting ( $r$ ) and end geometry (r-to-v) and then linearly interpolate the coordinates to sample nine structures in between. For scenario A, we first perform a constrained DFT geometry relaxation for the molecule adsorbed on the $r$ position in which we keep the atoms of the gold surface fixed and only allow the molecule to adjust (r-fix position). Since the molecule stays in this local minimum instead of sliding down into the valley, we perform a similar constrained DFT geometry relaxation with the molecule placed on the v position ( $\mathrm{v}$-fix position). We then take the optimized geometries of the molecule on the r-fix and v-fix position and perform again a linear interpolation of their coordinates. At each of these intermediate structures for pathways I and II we perform single-point DFT calculations without further structure relaxation to obtain the binding energy of $\alpha-6 \mathrm{~T}$ (for more details, please see the Supplemental Material [37]).

The resulting energy curves are presented in Figs. 3(a) and 3(b). Pathway I exhibits no energy barrier. The surface and molecule gain energy by deforming the gold surface. This process is self-propelled and does not need to be activated. Conversely, sliding the molecule down into the valley costs an initial energy of $\sim 0.3 \mathrm{eV}$. Once this barrier is overcome, the molecule gains a total of $0.44 \mathrm{eV}$ when it reaches the valley. This is much less than the $\sim 2.0 \mathrm{eV}$ gained in pathway I, indicating that the gold surface will relax at endpoint r-to-v to accommodate the arrival of the molecule. The lateral diffusion of the molecule is certainly a possible alternative process, but we expect it to be kinetically hindered at low temperatures and accompanied by a response of the gold surface akin to pathway $\mathrm{I}$ in scenario B.

Our DFT picture of an energetic preference for $\alpha-6 \mathrm{~T}$ in a valleylike adsorption environment agrees with experimental findings for $\alpha-6 \mathrm{~T}$ adsorption at very low coverages [0.015 monolayers (ML)]. The STM image depicted in Fig. 4(a) illustrates that the $\alpha-6 \mathrm{~T}$ molecules, represented as featureless, bright rods, lie well separated from each other and exclusively in valley positions.

A possible reason for the $\mathrm{v}$-position preference over the $r$ position could be charge transfer. Since the initial ridge position is not stable against further surface deformation, we fixed the surface atoms to compare the charge transfer upon $\alpha-6 \mathrm{~T}$ adsorption on these two positions. The Mulliken charge difference associated with adsorption on the r-fix and v-fix position is visualized in Fig. 3(c). In both cases, the surface has a higher negative charge after adsorption, most notably around the molecule, as indicated by the blue, ringlike pattern. Moreover, the sulfur atoms become positively charged 


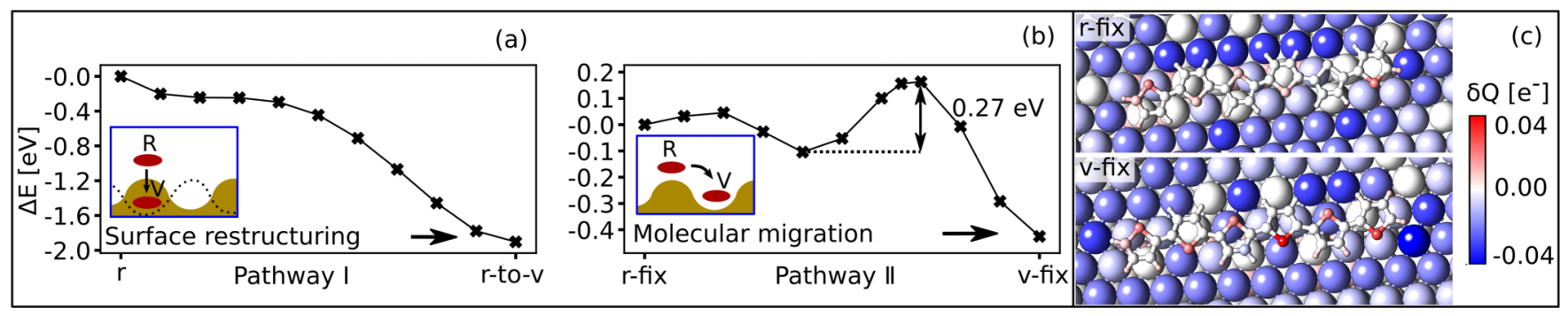

FIG. 3. Potential energy interpolation for pathways I and II. (a) In pathway I, the molecule creates its own valley. (b) In pathway II, the molecule slides off a gold ridge, whose structure is frozen. (c) Color-coded visualization of the Mulliken charge difference for the starting structure on ridge of the fixed surface (r-fix position) and the final structure in valley of the fixed surface (v-fix position). For the molecule adsorbed on the r-fix position, a smaller magnitude of charge transfer is found, as visible in the brighter red color of the sulfur atoms that corresponds to a lower positive charge.

after adsorption, indicating that the electron transfer from the molecule to the surface proceeds mainly by depleting sulfur orbitals with aromatic carbons being less affected. The total charge transfer from the molecule to the surface amounts to $0.40 e^{-}$for the molecule on the $\mathrm{v}$-fix position, but only $0.32 e^{-}$ for the molecule on the r-fix position. Hence, charge transfer is $20 \%$ reduced for the adsorption on the r-fix position, which can also be inferred from the lighter red color of the sulfur atoms. This smaller magnitude of charge transfer is accompanied by larger adsorption distances of the sulfur (3.47 $\AA$ vs $3.31 \AA$ for the r-fix position and the v-fix position) and a less planar conformation of the molecule on the $r$ site of the fixed surface, as expressed by the smaller median torsion angles of $169^{\circ}$ (see Table I).

Next, we compare theory and experiment. While in experiment organic film formation can be viewed as a sequential process of more and more molecules arriving on the gold surface, DFT calculations are constrained to a finite coverage due to periodic boundary conditions. In our case, one molecule per $5 \times 20$ unit cell amounts to a coverage of $24 \%$. When applying periodic boundary conditions, the lowenergy pathway (I) of an adsorption-induced displacement of the reconstruction ridges becomes a concerted shift of the complete $\mathrm{Au}$ reconstruction pattern across the (periodically treated) surface. In contrast, in experiment the adsorption of a single molecule is a nonperiodic event. Any adsorptioninduced ridge displacements will be local to the adsorption

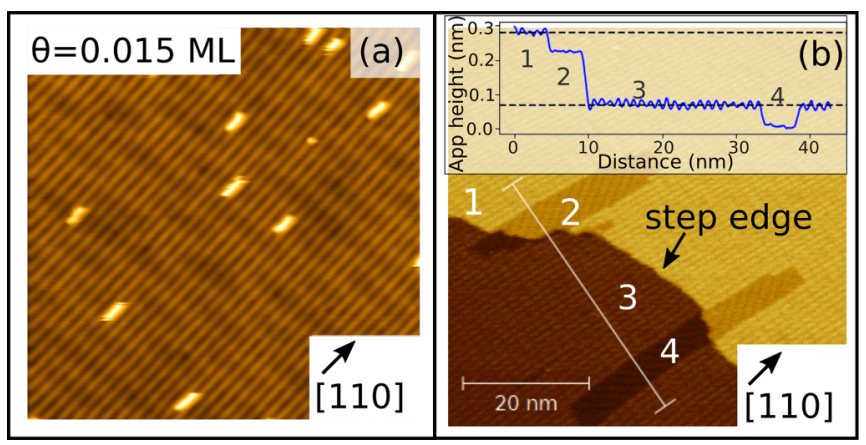

FIG. 4. (a) Low coverage adsorption of $\alpha-6 \mathrm{~T}$ on $\mathrm{Au}(100)$. The molecules adsorb exclusively in valley positions. (b) $\alpha-6 \mathrm{~T}$ monolayer growth on two substrate terraces. Two regions (2 and 4) with different adsorption morphologies can be distinguished on each terrace. The inset depicts the apparent height scan along the white line in (b). site of the molecule. Such local distortions will create defects such as stacking faults or domain walls further afield. The formation of these defects will cost energy that would have to be added to the adsorption energy of a single molecule for pathway I (scenario B) in a more complete model of the reconstruction process. The fact that our STM images [Fig. 4(a) for 0.014-ML coverage] do not reveal any local ridge distortions does not rule out their presence, but it indicates that the additional energy cost of ridge distortion and defect creation may add a barrier to pathway I that exceeds that of pathway II (sliding off the ridge). This finding may change with molecule size. We deduce that for the adsorption of longer oligothiophene molecules ( $\alpha$-nT with $n>6$ ) ridge displacements will become favorable, since the gain in relaxation energy scales linearly with molecular size, while the defect creation likely scales sublinearly. Our assumption is supported by the recent report of a rigid ridge shift around a single adsorbed polymer molecule for poly-3-hexyl-thiophene on $\mathrm{Au}(001)$ [46].

Another route towards overcoming the energy barrier for defect formation is to increase the molecular coverage to a dense monolayer. In that case, the concerted action of the molecules in self-assembled domains can even accomplish a temperature-facilitated partial lifting of the surface reconstruction as observed in STM measurements conducted at elevated temperatures [14].

Figure 4(b) depicts a large-scale STM image of $\alpha-6 \mathrm{~T}$ at monolayer coverage at $387 \mathrm{~K}$. Aside from large monolayer domains separated by a substrate step edge (areas 1 and 3), a stripelike smaller region exhibits a different structure on each substrate terrace (areas 2 and 4). The inset in Fig. 4(b) depicts the apparent height profile of the domains extracted along the white line. It shows that the molecules in the two stripes 2 and 4 are approximately $0.07 \mathrm{~nm} \pm 0.01 \mathrm{~nm}$ lower in height than the molecules in the large domains that are on the same substrate terrace, i.e., regions 1 and 3, respectively. $0.07 \mathrm{~nm}$ is exactly the aforementioned corrugation height of the reconstructed $\mathrm{Au}(100)$ surface compared to the unreconstructed $\mathrm{Au}(100)$ surface. This strongly indicates that in areas 2 and 4 the $\alpha-6 \mathrm{~T}$ molecules have modified the gold surface and lifted reconstruction. Moreover, the $\alpha-6 \mathrm{~T}$ film structure in areas 2 and 4 significantly differs from that in areas 1 and 3 . In both structures, $\alpha-6 \mathrm{~T}$ molecules are packed in molecular rows, but in areas 1 and 3 the long molecular axis of the molecules is oriented almost perpendicular to the direction of 
the molecular rows, whereas in areas 2 and 4 it is at a $30^{\circ}$ angle [14]. This rotated structure strongly resembles the adsorption pattern of $\alpha-6 \mathrm{~T}$ on $\operatorname{Ag}(100)$ [47], a surface that does not have any reconstruction.

Moreover, for the adsorption of styrene on $\mathrm{Au}(111)$ an adsorption-induced modification of the periodicity in the $\mathrm{Au}(111)$ reconstruction even at very low temperatures was proposed [12]. An extension of the surface herringbone reconstruction was also observed for the physisorption of dense layers of $\alpha-6 \mathrm{~T}$ [15] as well as Azure-A [13] on $\mathrm{Au}(111)$; in the latter case this effect was traced back to the cationic nature of the adsorbate, which enables stronger molecule-surface interactions combined with the presence of chloride anions that are known to affect the reconstruction of gold surfaces [48]. These findings suggest that the unusual behavior of $\alpha-6 \mathrm{~T}$ in $\mathrm{Au}(100)$ is not unique, but could be generalized to other, weakly adsorbing molecules.

In this Rapid Communication, we present a comprehensive first-principles investigation of aromatic oligomers on large, reconstructed Au surfaces. Our results reveal that $\alpha-6 \mathrm{~T}$ does not move off energetically ill-favored on-ridge positions, but instead moves the ridge out of the way to dig itself a preferred valley. While for weak adsorbates metal surfaces have been seen as immutable constraints that could be used to stir molecular adsorption, our findings illustrate that the picture is more complicated. The Au surface must instead be understood as soft and deformable, even by physisorbed molecules, and the notion of distinct adsorption sites does not apply.

This work was supported by the Deutsche Forschungsgemeinschaft (SFB TRR 102, B2), and by the Academy of Finland through its Centres of Excellence Programme (20152017) under Project No. 284621. The work has been performed under the Project HPC-EUROPA3 (INFRAIA-20161-730897), with the support of the EC Research Innovation Action under the H2020 Programme; in particular, the authors gratefully acknowledge the computer resources and technical support provided by the CSC-IT Center for Science and the Aalto Science-IT Project. D.G. acknowledges financial support by the Swiss National Science Foundation under Project No. P2ZHP2 168444.
[1] J. Gong, Chem. Rev. 112, 2987 (2012).

[2] Y. Zhang, X. Cui, F. Shi, and Y. Deng, Chem. Rev. 112, 2467 (2012).

[3] X. Zhang and Y. Ding, Catal. Sci. Technol. 3, 2862 (2013).

[4] H. Masatake, Angew. Chem., Int. Ed. 53, 52 (2014).

[5] A. Wittstock and M. Bäumer, Acc. Chem. Res. 47, 731 (2014).

[6] P. Maksymovych, D. C. Sorescu, and J. T. Yates, Phys. Rev. Lett. 97, 146103 (2006).

[7] A. Cossaro, R. Mazzarello, R. Rousseau, L. Casalis, A. Verdini, A. Kohlmeyer, L. Floreano, S. Scandolo, A. Morgante, M. L. Klein, and G. Scoles, Science 321, 943 (2008).

[8] G. Nenchev, B. Diaconescu, F. Hagelberg, and K. Pohl, Phys. Rev. B 80, 081401(R) (2009).

[9] P. Maksymovych, O. Voznyy, D. B. Dougherty, D. C. Sorescu, and J. T. Yates, Prog. Surf. Sci. 85, 206 (2010).

[10] I. Chizhov, A. Kahn, and G. Scoles, J. Cryst. Growth 208, 449 (2000).

[11] W. Zhao, H. Zhu, H. Song, J. Liu, Q. Chen, Y. Wang, and K. Wu, J. Phys. Chem. C 122, 7695 (2018).

[12] A. E. Baber, S. C. Jensen, E. V. Iski, and E. C. H. Sykes, J. Am. Chem. Soc. 128, 15384 (2006).

[13] F. Rossel, P. Brodard, F. Patthey, N. V. Richardson, and W.-D. Schneider, Surf. Sci. 602, L115 (2008).

[14] A. Höfer, K. Duncker, M. Kiel, S. Wedekind, and W. Widdra, Phys. Rev. B 83, 075414 (2011).

[15] M. Kiel, K. Duncker, C. Hagendorf, and W. Widdra, Phys. Rev. B 75, 195439 (2007).

[16] D. G. Fedak and N. A. Gjostein, Phys. Rev. Lett. 16, 171 (1966).

[17] M. V. Hove, R. Koestner, P. Stair, J. Bibérian, L. Kesmodel, I. Bartô̂, and G. Somorjai, Surf. Sci. 103, 189 (1981).

[18] R. Hammer, A. Sander, S. Förster, M. Kiel, K. Meinel, and W. Widdra, Phys. Rev. B 90, 035446 (2014).

[19] W. Moritz and D. Wolf, Surf. Sci. 88, L29 (1979).

[20] D. dos Reis, F. Negreiros, V. de Carvalho, and E. Soares, Surf. Sci. 604, 568 (2010).
[21] C. Wöll, S. Chiang, R. J. Wilson, and P. H. Lippel, Phys. Rev. B 39, 7988 (1989).

[22] J. V. Barth, H. Brune, G. Ertl, and R. J. Behm, Phys. Rev. B 42, 9307 (1990).

[23] A. Kühnle, L. M. Molina, T. R. Linderoth, B. Hammer, and F. Besenbacher, Phys. Rev. Lett. 93, 086101 (2004).

[24] F. Hiebel, B. Shong, W. Chen, R. J. Madix, E. Kaxiras, and C. M. Friend, Nat. Commun. 7, 13139 (2016).

[25] A. D. Jewell, H. L. Tierney, and E. C. H. Sykes, Phys. Rev. B 82, 205401 (2010).

[26] N. Koch, ChemPhysChem 8, 1438 (2007).

[27] S. Günes, H. Neugebauer, and N. S. Sariciftci, Chem. Rev. 107, 1324 (2007).

[28] S. Steinberger, A. Mishra, E. Reinold, J. Levichkov, C. Uhrich, M. Pfeiffer, and P. Bauerle, Chem. Commun. 47, 1982 (2011).

[29] G. Horowitz, D. Fichou, X. Peng, Z. Xu, and F. Garnier, Solid State Commun. 72, 381 (1989).

[30] G. Horowitz, Adv. Mater. 2, 287 (1990).

[31] A. Dodabalapur, L. Torsi, and H. E. Katz, Science 268, 270 (1995).

[32] B. S. Ong, Y. Wu, P. Liu, and S. Gardner, J. Am. Chem. Soc. 126, 3378 (2004).

[33] E. M. Mannebach, J. W. Spalenka, P. S. Johnson, Z. Cai, F. J. Himpsel, and P. G. Evans, Adv. Funct. Mater. 23, 554 (2013).

[34] V. Blum, R. Gehrke, F. Hanke, P. Havu, V. Havu, R. Xinguo, K. Reuter, and M. Scheffler, Comput. Phys. Commun. 180, 2175 (2009).

[35] J. P. Perdew, K. Burke, and M. Ernzerhof, Phys. Rev. Lett. 77, 3865 (1996).

[36] A. Tkatchenko and M. Scheffler, Phys. Rev. Lett. 102, 073005 (2009).

[37] See Supplemental Material at http://link.aps.org/supplemental/ 10.1103/PhysRevMaterials.3.011601 for a comparison of the TS vdw and the vdW ${ }^{\text {surf }}$ method for $\alpha-6 \mathrm{~T}$ on the reconstructed $\mathrm{Au}(100)$ surface, which includes Refs. [36,49-53]. 
[38] C. van Wüllen, J. Chem. Phys. 109, 392 (1999).

[39] J. van Lenthe, S. Faas, and J. Snijders, Chem. Phys. Lett. 328, 107 (2000).

[40] P. Havu, V. Blum, V. Havu, P. Rinke, and M. Scheffler, Phys. Rev. B 82, 161418 (2010).

[41] P. Heilmann, K. Heinz, and K. Müller, Surf. Sci. 83, 487 (1979).

[42] P. W. Palmberg and T. N. Rhodin, Phys. Rev. 161, 586 (1967).

[43] R. S. Mulliken, J. Chem. Phys. 23, 1833 (1955).

[44] E. C. Beret, L. M. Ghiringhelli, and M. Scheffler, Faraday Discuss. 152, 153 (2011).

[45] H. Jónsson, G. Mills, and K. W. Jacobsen, Classical and Quantum Dynamics in Condensed Phase Simulations, edited by B. J. Berne, G. Ciccotti, and D. F. Coker (World Scientific, Singapore, 1998), pp. 385-404.
[46] S. Förster and W. Widdra, J. Chem. Phys. 141, 054713 (2014).

[47] K. Duncker, M. Kiel, A. Höfer, and W. Widdra, Phys. Rev. B 77, 155423 (2008)

[48] D. Kolb, Prog. Surf. Sci. 51, 109 (1996).

[49] V. G. Ruiz, W. Liu, E. Zojer, M. Scheffler, and A. Tkatchenko, Phys. Rev. Lett. 108, 146103 (2012).

[50] E. M. Lifshitz, Sov. Phys. JETP 2, 73 (1956).

[51] E. Zaremba and W. Kohn, Phys. Rev. B 13, 2270 (1976).

[52] A. Kumar, K. Banerjee, M. Dvorak, F. Schulz, A. Harju, P. Rinke, and P. Liljeroth, ACS Nano 11, 4960 (2017).

[53] R. J. Maurer, V. G. Ruiz, J. Camarillo-Cisneros, W. Liu, N. Ferri, and K. Reuter, and A. Tkatchenko, Prog. Surf. Sci. 91, 72 (2016). 